\title{
Accuracy of the IASLC/ATS/ERS histological subtyping of stage I lung adenocarcinoma on intraoperative frozen sections
}

\author{
Humberto E Trejo Bittar ${ }^{1}$, Pimpin Incharoen ${ }^{1}$, Andrew D Althouse ${ }^{2}$ and Sanja Dacic ${ }^{1}$ \\ ${ }^{1}$ Department of Pathology, University of Pittsburgh Medical Center, Pittsburgh, PA, USA and ${ }^{2}$ Department of \\ Obstetrics, Gynecology, and Reproductive Science, Magee Womens Research Institute, University of \\ Pittsburgh, Pittsburgh, PA, USA
}

\begin{abstract}
Histological subtyping of surgically resected lung adenocarcinoma has been shown to be of prognostic significance, and limited surgical resection has been proposed as a treatment of choice for early-stage lung adenocarcinoma. The accuracy of histological subtyping has been recently assessed in the surgical resection and small biopsy specimens; however, the accuracy of intraoperative subtyping on frozen sections remains relatively unknown. The aim of this study was to determine diagnostic accuracy and interobserver variability in histological subtyping of lung adenocarcinoma on intraoperative frozen sections. Overall, 112 consecutive cases of surgically resected stage I lung adenocarcinoma were reviewed independently by three pathologists. Histological patterns (acinar, lepidic, papillary, micropapillary, and solid) and mucinous variant were recorded in $5 \%$ increments for each intraoperative frozen and permanent sections. Primary and secondary histological patterns were assigned in each case. Kappa scores were calculated to evaluate agreement between pathologists in the assessment of histological subtype on intraoperative frozen sections versus permanent sections. Overall agreement between intraoperative frozen and permanent sections was moderate for primary pattern $(69.7 \%$ of cases), with kappa scores ranging from 0.43 to 0.58 , with more consistent agreement for stage IA tumors. Kappa scores for the secondary pattern ranged from 0.16 to 0.32 . Acinar and solid patterns were most likely to be correctly identified as primary growth patterns. Micropapillary pattern was recognized in only $11-55 \%$ of cases. The main reasons for discrepancies between intraoperative frozen and permanent sections were inadequate sampling and poor quality of frozen sections. Our study suggests that it is difficult to predict the primary adenocarcinoma pattern on a single representative frozen section. This observation suggests a potential impact on the extent of frozen section sampling by pathologists at the time of intraoperative consultation, if surgical management of stage I lung adenocarcinoma will be guided by its histological subtype.
\end{abstract}

Modern Pathology (2015) 28, 1058-1063; doi:10.1038/modpathol.2015.71; published online 29 May 2015

Many studies reported prognostic predictors and risk factors for recurrence in surgically resected nonsmall cell lung carcinoma including older age, male gender, angiolymphatic invasion, and tumor size. ${ }^{1-3}$ The prognostic significance of histological subtyping of lung adenocarcinoma has been demonstrated in several studies and histological subtyping in 5\% increments of the surgically resected lung adenocarcinoma has been recommended by the International Association for the Study of Lung Cancer (IASLC),

Correspondence: Dr HE Trejo Bittar, MD, Department of Pathology, University of Pittsburgh Medical Center, PUH-A610, 200 Lothrop Street, Pittsburgh, PA 15213-2582, USA.

E-mail: trejobittarhe@upmc.edu and htotbittar@gmail.com

Received 12 February 2015; revised 5 May 2015; accepted 6 May 2015; published online 29 May 2015 the American Thoracic Society (ATS), and the European Respiratory Society (ERS) classification of lung adenocarcinoma, and most recently by the 2015 WHO classification of tumors of the lung. ${ }^{4-8}$ Solid and micropapillary patterns have been associated with poor outcome in stage I lung adenocarcinoma, whereas tumors with lepidic growth pattern showed favorable outcome. ${ }^{5,9-12}$ Mucinous morphology has also been reported as a negative prognostic indicator. ${ }^{13}$ More recently, it has been recognized that adenocarcinoma with more than $10 \%$ cribriform component is associated with high risk of recurrence. ${ }^{14}$

The type of surgical resection for peripheral, earlystage lung adenocarcinoma remains controversial, and lobectomy with lymph node dissection is still the most common approach. ${ }^{15}$ Some surgeons have 
suggested that small (usually less than $2 \mathrm{~cm}$ ) peripheral lung adenocarcinoma can be treated with a limited resection with survival and recurrence rate similar to lobectomy. ${ }^{16-18}$ Recently, Nitadori et al ${ }^{10}$ reported that the presence of $5 \%$ or greater of micropapillary component in lung adenocarcinoma measuring up to $2 \mathrm{~cm}$ in diameter and treated with limited surgical resection was associated with an increased risk of recurrence. This observation has potential practical implications for pathologists, who have to make an effort to accurately recognize histological patterns on the frozen sections. However, the accuracy of histological subtyping on frozen sections is relatively unexplored, and to our knowledge it was addressed in a single study only. ${ }^{19}$

The aim of this study was to determine diagnostic accuracy and interobserver variability in histological subtyping of lung adenocarcinoma on frozen sections obtained at the time of intraoperative consultation.

\section{Materials and methods}

\section{Patient Selection}

A total of 112 consecutive surgically resected primary-stage IA/IB invasive lung adenocarcinoma specimens, with hematoxylin and eosin (H\&E)stained frozen and permanent sections available for review, were identified in the archives of the University of Pittsburgh Medical Center Department of Pathology. Specimens included 24 wedge resections, 30 segmentectomies, 55 lobectomies, and 3 pneumonectomies. There were 48 male patients and 64 female patients, with an age at diagnosis ranging from 41 to 89 years (mean 66). There were 77 patients with stage IA and 35 patients with stage IB tumors. The study was conducted under an exemption approved by the University of Pittsburgh Institutional Review Board (PRO 12070229).

\section{Review of H\&E-Stained Frozen and Permanent Sections}

All available $\mathrm{H} \& \mathrm{E}$-stained sections were independently reviewed by three pathologists including a senior pathologist in training (HETB), a general pathologist (PI), and a thoracic pathologist (SD). Histologic subtyping was performed according to the proposed IASLC/ATS/ERS lung adenocarcinoma classification and the 2015 World Health Organization (WHO) classification criteria. ${ }^{4,8}$ In brief, lepidic, acinar, papillary, micropapillary, and solid histologic patterns and mucinous variant were recorded in 5\% increments. The primary (predominant) histologic subtype was considered to be the one with the highest percentage.

\section{Statistical Analysis}

Kappa $(\kappa)$ scores were calculated to evaluate the agreement for each pathologist for the correct identification of the primary and secondary histologic patterns/variant. Discordant cases were identified as those with discrepancy for all three pathologists. Comparison of the agreement for stage IA and IB tumors was also evaluated. Interpretation of $\kappa$ scores was as follows: 0.81 and 1.0 as almost perfect agreement, $0.61-0.80$ as substantial agreement, $0.41-0.60$ as moderate agreement, $0.21-0.40$ as fair agreement, and $0-0.20$ as slight agreement. ${ }^{20}$ Statistical analyses were performed using SAS version 9.4 (SAS Institute, Cary NC, USA).

\section{Results}

\section{Histological Subtypes on Permanent Sections}

Table 1 summarizes histological patterns of 112 lung adenocarcinoma determined on the permanent sections. The predominant primary histologic subtype was acinar (59/112; 52.7\%), followed by solid $(17 / 112 ; 15.2 \%)$ and lepidic (16/112; 14.3\%) patterns. No micropapillary-predominant ADC was found. Twenty $(20 / 112 ; 17 \%)$ cases showed a single pattern. The most frequent secondary pattern was acinar $(36 / 92 ; 39.1 \%)$, followed by lepidic $(23 / 92$; $25 \%)$ and papillary $(19 / 92 ; 20.7 \%)$ patterns.

\section{Accuracy of Adenocarcinoma Histological Subtyping on Intraoperative Frozen Sections}

Table 2 summarizes the accuracy of histological subtyping of lung adenocarcinoma on intraoperative frozen sections. The primary histologic pattern was correctly identified on frozen sections in 78/112 $(69.7 \%)$ of cases. Overall agreement for the accuracy of histological subtyping on frozen sections was 'moderate' $(\kappa=0.51)$ ranging from $\kappa=0.43$ for pathologist in training to $\kappa=0.58$ for thoracic pathologist. Agreement was more consistent between pathologists for stage IA tumors than for stage IB tumors. For stage IA tumors, overall agreement was 'moderate' $(\kappa=0.52)$, ranging from $\kappa=0.49$ (general pathologist) to $\kappa=0.55$ (pathologist in training); however, there was much more variations in agreement for stage IB tumors, with an overall 'moderate' agreement $(\kappa=0.48)$, ranging from 'fair' agreement $(\kappa=0.29$

Table 1 Primary and secondary histologic patterns on permanent sections in 112 cases of lung adenocarcinoma

\begin{tabular}{lcc}
\hline Pattern & Primary (\%) & Secondary (\%) \\
\hline Acinar & $59(52.7)$ & $36(39.1)$ \\
Lepidic & $16(14.3)$ & $23(25)$ \\
Papillary & $14(12.5)$ & $19(20.7)$ \\
Micropapillary & $0(0)$ & $5(5.4)$ \\
Solid & $17(15.2)$ & $8(8.7)$ \\
Variant & & $1(1.1)$ \\
$\quad$ Mucinous & $6(5.4)$ & \\
\hline
\end{tabular}


for general pathologist) to 'substantial' agreement ( $\kappa=0.71$ for thoracic pathologist).

The primary histological patterns that were most likely to be correctly identified on frozen sections were acinar (sensitivity $87.6 \%$; specificity $65.4 \%$ ) and solid (sensitivity $72.6 \%$; specificity 95.8\%). Lepidic-predominant tumors showed low sensitivity $(33.3 \%)$ but very high specificity (95.4\%). Papillarypredominant tumors had better sensitivity $(50 \%)$ and also very high specificity (96.6\%). Mucinous tumors showed the lowest sensitivity $(27.8 \%)$ while specificity was the highest (98.4\%; Table 2).

The secondary histologic patterns were least likely to be correctly identified, with sensitivities ranging as low as $13.3 \%$ for micropapillary pattern to $50 \%$ for solid pattern. The specificity for the secondary histologic pattern was high for all patterns, ranging from $88.6 \%$ for the acinar pattern to $98.1 \%$ for the micropapillary pattern. Overall agreement for the secondary histologic pattern was 'fair' $(\kappa=0.25)$ and lower than that for the primary histologic pattern, with agreement ranging from 'slight' agreement ( $\kappa=0.16$ for general pathologist) to 'fair' agreement

Table 2 Accuracy of histological subtyping on intraoperative frozen sections

\begin{tabular}{lcc}
\hline Pattern & $\begin{array}{c}\text { Sensitivity, } \\
\%(95 \% \text { CI })\end{array}$ & $\begin{array}{c}\text { Specificity, } \\
\%(95 \% \text { CI })\end{array}$ \\
\hline $\begin{array}{l}\text { Primary pattern } \\
\text { Acinar }\end{array}$ & $57.6(81.4-93.7)$ & $65.4(56.5-74.3)$ \\
Lepidic & $33.3(8.5-58.2)$ & $94.4(88.6-100)$ \\
Papillary & $50(41.9-58.1)$ & $96.6(93.7-99.5)$ \\
Micropapillary & NA & NA \\
Solid & $72.6(51.1-94)$ & $95.8(94.6-97)$ \\
Mucinous variant & $27.8(0-56.6)$ & $98.4(96.2-100)$ \\
Secondary pattern & & \\
Acinar & $32.4(14-50.8)$ & $88.6(82.6-94.6)$ \\
Lepidic & $42(22.2-61.9)$ & $88.8(83.2-94.3)$ \\
Papillary & $31.6(15.8-47.3)$ & $91(82.9-99.1)$ \\
Micropapillary & $13.3(0.3-26.4)$ & $98.1(96-100)$ \\
Solid & $50(12.6-87.4)$ & $95.2(94.1-96.3)$ \\
Mucinous variant & NA & NA \\
\hline
\end{tabular}

Abbreviations: CI, confidence interval; NA: no data available.
( $\kappa=0.32$ for thoracic pathologist). Agreement was similar between stage IA and IB tumors; for stage IA tumors overall agreement was 'fair' $(\kappa=0.25)$, ranging from 'slight' agreement $(\kappa=0.16$ for general pathologist) to 'fair' agreement ( $\kappa=0.28$ for thoracic pathologist). For stage IB tumors, overall agreement was 'fair' $(\kappa=0.28)$, ranging from 'slight' agreement ( $\kappa=0.14$ for general pathologist) to 'fair' agreement $(\kappa=0.37$ for thoracic pathologist).

\section{Discordant Cases}

The predominant histologic pattern of 12 cases $(12 / 112,10.7 \%)$ was misinterpreted by all three study pathologists. These were mostly stage IA (nine cases) tumors, whereas the remaining three cases were stage IB tumors. Technical errors were the most common reason for discrepant interpretations, including four cases with a poor-quality section and another four cases with inadequate, very small amount of tumor tissue submitted at the time of intraoperative frozen section consultation (Figure 1). The histologic pattern that was most likely to be misinterpreted was lepidic (five cases), which in all cases was interpreted as acinar by all three pathologists (Figure 2). Two acinar-predominant tumors were interpreted as solid- and lepidic-predominant. Two papillary-predominant adenocarcinomas were interpreted as acinar- and lepidic-predominant, whereas two invasive mucinous adenocarcinomas were interpreted as papillary- and solid-predominant. One solid-predominant tumor was misclassified as acinar.

\section{Discussion}

Our study demonstrated that the accurate assessment of adenocarcinoma histologic subtype on frozen sections is challenging. We found a 'moderate' agreement for the primary pattern, and only a 'fair' agreement for the secondary pattern. Our findings are similar to that of Yeh et $a{ }^{19}{ }^{19}$ who reported unsatisfactory accuracy of frozen sections for histologic subtyping with also only 'moderate' agreement
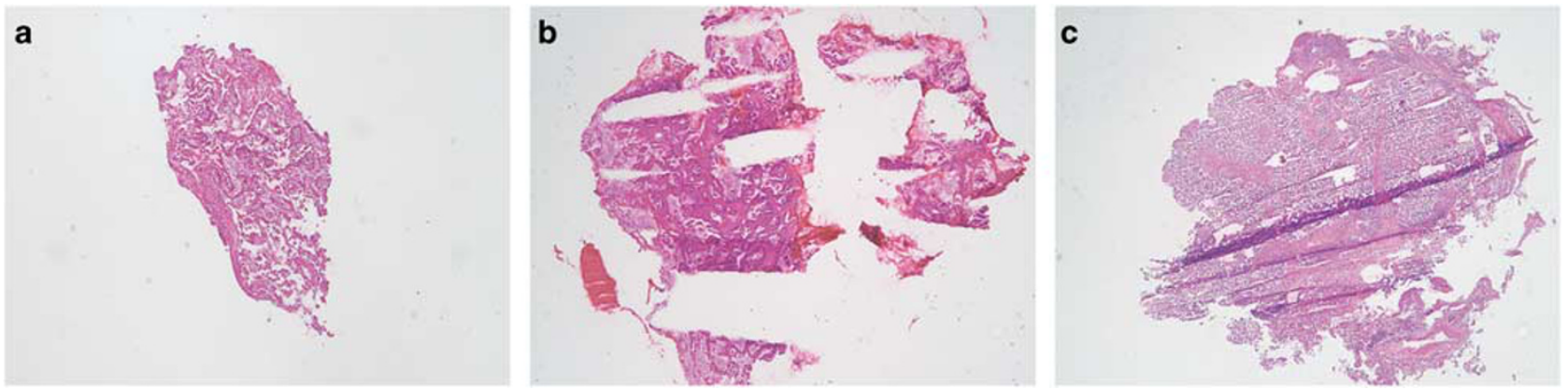

Figure 1 Examples of discordant cases with inadequate sampling and poor quality of frozen sections. Very small sampling of a 3-cm tumor $(\mathbf{a}$, hematoxylin and eosin $(\mathrm{H} \& \mathrm{E}), \times 40)$ and a $1.4-\mathrm{cm}$ tumor $(\mathbf{b}, \mathrm{H} \& \mathrm{E}, \times 20)$. A prominent frozen section artifact $(\mathbf{c}, \mathrm{H} \& \mathrm{E}, \times 20)$. 


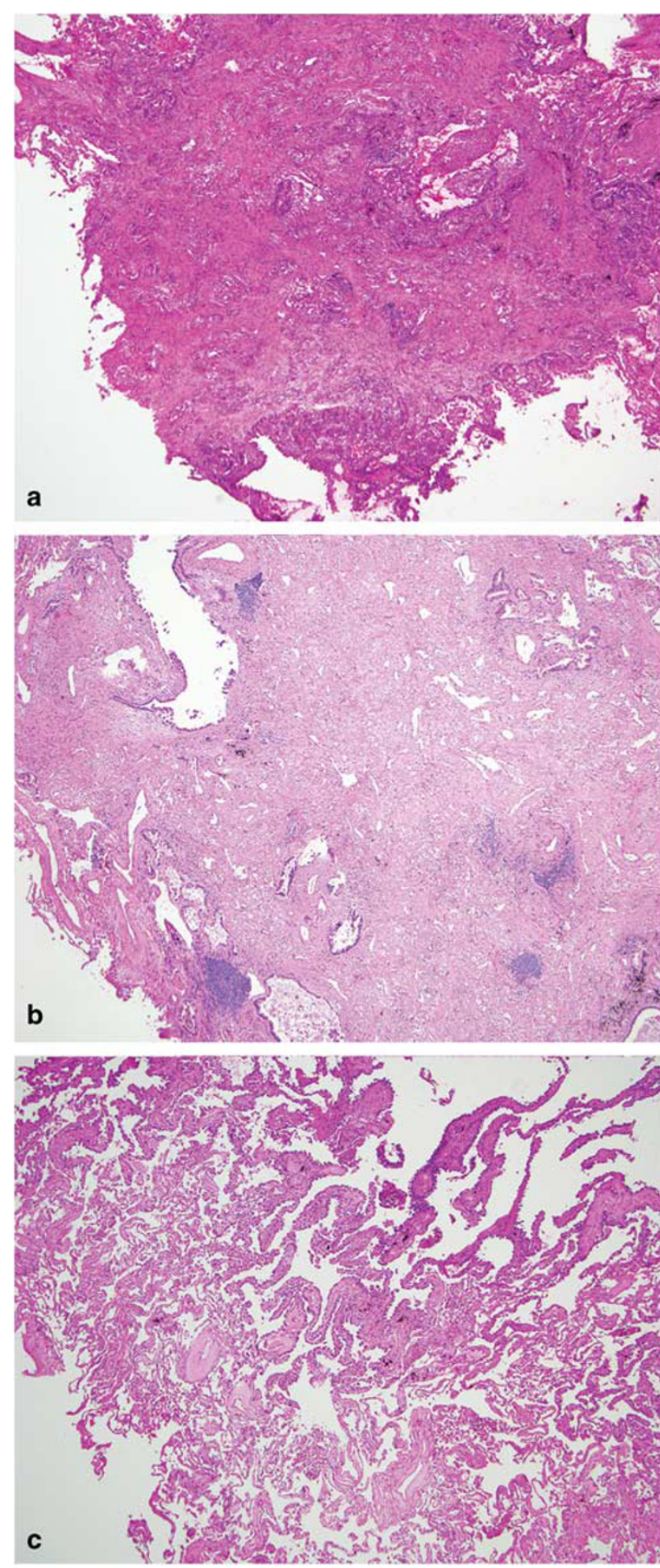

Figure 2 Lepidic pattern was most frequently misinterpreted because of suboptimal sampling. Frozen section interpreted as acinar-predominant (a, hematoxylin and eosin (H\&E), $\times 40)$; a permanent section of the same area $(\mathbf{b}, \mathrm{H} \& \mathrm{E}, \times 40)$; the entirely submitted tumor showed a lepidic-predominant pattern (c, H\&E, $\times 100)$. $(\kappa=0.565)$. Both studies are in contrast to the study by Motoi et al, ${ }^{21}$ who reported $98.6 \%$ accuracy in histological subtyping. Studies performed to evaluate accuracy and agreement on permanent sections have shown much better 'substantial' agreement for conventional cases $(\kappa=0.77)$, whereas agreement for difficult cases was only 'fair' $(\kappa=0.38){ }^{22}$

Some histologic patterns were more difficult to predict, with micropapillary, mucinous, and lepidic being the most challenging to identify. Sensitivity and specificity data for each histologic subtype provided relevant information about the difficulty to identify certain histologic patterns. Acinar and solid patterns were most likely to be correctly identified on frozen sections, as these patterns showed the highest sensitivity, $87.6 \%$ and $72.6 \%$, respectively, with a very high specificity for the solid-predominant tumors $(95.8 \%)$. This is similar to results reported by Yeh at al. ${ }^{19}$ Interestingly, lepidicpredominant tumors showed a low sensitivity in our study $(33.3 \%)$ and a very high specificity (94.4\%). This result might be related to the fact that lepidicpredominant tumors showed a combination of acinar and lepidic patterns on the frozen sections and therefore were most frequently misclassified as acinar-predominant. However, after the entire tumor was submitted for permanent sections, the lepidicpredominant pattern was present indicating sampling error. The possibility of interpretation error should also be considered in some cases. Thunnissen et $a l^{22}$ reported difficulties in differentiating acinar, papillary, and lepidic patterns on permanent sections. Authors suggested that cross-cutting of tumor growing along alveolar septae (lepidic) could mimic papillary pattern, which in our study sample showed a relatively low sensitivity (50\%). Furthermore, authors indicated that desmoplastic stromal reaction could be mimicked by the collapsed lepidic pattern, a feature that would be very hard to recognize on frozen sections. In the current study, the most common reason for misclassification of the lepidic pattern was incomplete tumor sampling rather than interpretation error. Another possible explanation could be related to the fact that frozen sections were not performed on inflated lungs, as collapsed alveolar spaces can make interpretation of lepidic pattern extremely difficult. ${ }^{23}$ One of the limitations of our study was the lack of tumors with a primary micropapillary pattern. However, cases with a secondary micropapillary pattern showed extremely low sensitivity (13\%), but a very high specificity $(98 \%)$. Yeh et $a l^{19}$ reported similar observation for the micropapillary primary pattern. Nitadori et $a l^{10}$ reported a high recurrence rate in adenocarcinoma with micropapillary pattern treated with limited resections. If this observation is to be confirmed by other studies, then it is very likely that micropapillary adenocarcinoma will be treated by lobectomy. The high specificity for its identification on frozen sections is reassuring that these tumors most likely will be recognized on adequately 
sampled tumors at the time of intraoperative consultation.

Our study provides an interesting observation regarding the stage of tumor (IA versus IB), which can be translated to tumor size. We found a larger variability for the degree of agreement between pathologists for the larger stage IB tumors. This finding suggests that the sampling error was most likely the reason for inaccurate subtyping, as larger tumors were more difficult to correctly subtype. Interestingly, smaller tumors (stage IA) were more often misclassified than larger tumors in the difficult cases (9/12), which suggest that factors other than sampling error also have a role in the misinterpretation of cases on frozen sections including pathologist's experience, interpretation errors, and poor quality of frozen sections. Similarly, Yeh et al ${ }^{19}$ also found sampling error to be the main reason for the disagreement between frozen and permanent sections. The fact that secondary pattern showed overall less agreement also supports sampling error as one of the reasons for the disagreement.

Our study suggests that the pathologist's experience may not be the main factor that determines accurate histological subtyping of lung adenocarcinoma on frozen sections. Only the agreement of the primary pattern on stage IB tumor showed significant variability between pathologists, with the thoracic pathologist showing a 'substantial' agreement. However, pathologist regardless of experience showed comparable 'moderate' agreement for the primary pattern in stage IA tumors. In contrast, Warth et al ${ }^{24}$ reported that thoracic pathologists showed 'substantial' agreement for the identification of predominant pattern on permanent sections, in contrast to pathologist in training with only 'fair' agreement. Possible reasons for this discordance could be related to different proportion of predominant histologic patterns between both study samples (our sample included more acinar- and papillarypredominant tumors and less lepidic- and solidpredominant tumors) and the fact that Warth et $a l^{24}$ used permanent sections only.

Our study has some limitations. First, no survival data were provided. This was carried out intentionally, as our main focus was to determine the pathologist's ability to accurately determine the histological pattern of lung adenocarcinoma. Furthermore, this study is retrospective and all tumors had only one representative section submitted for frozen section, and therefore survival analysis would not reflect the biology of disease. Finally, we observed large differences in the quality of frozen sections that were prepared by different pathologists in training, pathology assistants, and pathologists with different levels of technical skills in preparation of frozen sections and at different time points.

In summary, our data indicate that subtyping of lung adenocarcinoma on frozen sections is difficult and may not be reliable. The reasons for the low agreement are multiple, but mainly related to sampling artefact of larger and more heterogeneous tumors, the quantity of the tumor tissue submitted for frozen sections and quality of the frozen section slides. If limited surgical resection will become a new standard of care, the role of surgical pathologists in the intraoperative assessment of histological subtype of lung adenocarcinoma, especially those associated with poor outcome, will become extremely important in surgical decision making. Therefore, studies that would provide the recommendations for practice improvement are desired.

\section{Disclosure/conflict of interest}

The authors declare no conflicts of interest.

\section{References}

1 Ravdin PM, Davis G. Prognosis of patients with resected non-small cell lung cancer: impact of clinical and pathologic variables. Lung Cancer 2006;52:207-212.

2 Yamato Y, Koike T, Yoshiya K et al. Results of surgical treatment for small ( $2 \mathrm{~cm}$ or under) adenocarcinomas of the lung. Surg Today 2008;38:109-114.

3 Maeda R, Yoshida J, Ishii G et al. Poor prognostic factors in patients with stage IB non-small cell lung cancer according to the seventh edition TNM classification. Chest 2011;139:855-861.

4 Travis WD, Brambilla E, Noguchi M et al. International association for the study of lung cancer/american thoracic society/european respiratory society international multidisciplinary classification of lung adenocarcinoma. J Thorac Oncol 2011;6:244-285.

5 Yoshizawa A, Motoi N, Riely GJ et al. Impact of proposed IASLC/ATS/ERS classification of lung adenocarcinoma: prognostic subgroups and implications for further revision of staging based on analysis of 514 stage I cases. Mod Pathol 2011;24:653-664.

6 Okada M. Subtyping lung adenocarcinoma according to the novel 2011 IASLC/ATS/ERS classification: correlation with patient prognosis. Thorac Surg Clin 2013;23: 179-186.

7 Warth A, Muley T, Meister M et al. The novel histologic International Association for the Study of Lung Cancer/ American Thoracic Society/European Respiratory Society classification system of lung adenocarcinoma is a stage-independent predictor of survival. J Clin Oncol 2012;30:1438-1446.

8 Travis WD, Noguchi M, Yatabe Y et al. AdenocarcinomaIn:Travis WD, Brambilla E, Burke AP, Marx A, Nicholson AG(eds) WHO Classification of Tumours of the Lung, Pleura, Thymus and Heart 4th (edn.) Vol. 7 IARC: Lyon 2015, pp 26-36.

9 Dembitzer FR, Flores RM, Parides MK, Beasley MB. Impact of histologic subtyping on outcome in lobar vs sublobar resections for lung cancer: a pilot study. Chest 2014;146:175-181.

10 Nitadori J, Bograd AJ, Kadota K et al. Impact of micropapillary histologic subtype in selecting limited resection vs lobectomy for lung adenocarcinoma of 2cm or smaller. J Natl Cancer Inst 2013;105:1212-1220.

11 Sanchez-Mora N, Presmanes MC, Monroy V et al. Micropapillary lung adenocarcinoma: a distinctive 
histologic subtype with prognostic significance. Case series. Hum Pathol 2008;39:324-330.

12 Ohtaki Y, Yoshida J, Ishii G et al. Prognostic significance of a solid component in pulmonary adenocarcinoma. Ann Thorac Surg 2011;91:1051-1057.

13 Marchetti A, Buttitta F, Pellegrini S et al. Bronchioloalveolar lung carcinomas: K-ras mutations are constant events in the mucinous subtype. J Pathol 1996;179: 254-259.

14 Kadota K, Yeh YC, Sima CS et al. The cribriform pattern identifies a subset of acinar predominant tumors with poor prognosis in patients with stage I lung adenocarcinoma: a conceptual proposal to classify cribriform predominant tumors as a distinct histologic subtype. Mod Pathol 2014;27:690-700.

15 Howington JA, Blum MG, Chang AC et al. Treatment of stage I and II non-small cell lung cancer: diagnosis and management of lung cancer, 3rd ed: American College of Chest Physicians evidence-based clinical practice guidelines. Chest 2013;143:e278S-e313S.

16 Carr SR, Schuchert MJ, Pennathur A et al. Impact of tumor size on outcomes after anatomic lung resection for stage $1 \mathrm{~A}$ non-small cell lung cancer based on the current staging system. J Thorac Cardiovasc Surg 2012;143:390-397.

17 Koike T, Koike T, Yamato Y et al. Prognostic predictors in non-small cell lung cancer patients undergoing intentional segmentectomy. Ann Thorac Surg 2012;93: 1788-1794.
18 Landreneau RJ, Normolle DP, Christie NA et al. Recurrence and survival outcomes after anatomic segmentectomy versus lobectomy for clinical stage I non-small-cell lung cancer: a propensity-matched analysis. J Clin Oncol 2014;32:2449-2455.

19 Yeh YC, Nitadori JI, Kadota K et al. Using frozen section to identify histologic patterns in stage I lung adenocarcinoma $</=3 \mathrm{~cm}$ : accuracy and interobserver agreement. Histopathology 2015;66:922-938.

20 Landis JR, Koch GG. The measurement of observer agreement for categorical data. Biometrics 1977;33: 159-174.

21 Motoi N, Hamanaka W, Oba $\mathrm{T}$ et al. Evaluation of histologic accuracy on diagnosis and inasion of smallsized lung cancer usin intra-operative frozen section. J Thorac Oncol 2011;6:S566.

22 Thunnissen E, Beasley MB, Borczuk AC et al. Reproducibility of histopathological subtypes and invasion in pulmonary adenocarcinoma. An international interobserver study. Mod Pathol 2012;25:1574-1583.

$23 \mathrm{Xu} \mathrm{X}$, Chung JH, Jheon S et al. The accuracy of frozen section diagnosis of pulmonary nodules: evaluation of inflation method during intraoperative pathology consultation with cryosection. J Thorac Oncol 2010;5: 39-44.

24 Warth A, Stenzinger A, von Brunneck AC et al. Interobserver variability in the application of the novel IASLC/ATS/ERS classification for pulmonary adenocarcinomas. Eur Respir J 2012;40:1221-1227. 